\title{
A FORMAL DEFINITION OF ALGOL 60 AS DESCRIBED IN THE 1975 MODIFIED REPORT
}

Wolfgang Henhapl \& Cliff B.Jones

\author{
Abstraat: \\ This paper provides a formal definition of a version \\ of the ALGOL 60 programing language. In particular \\ the definition uses the denotational approach and the \\ meta-language presented in this volume (-- known with= \\ in the Vienna Laboratory as "META-IV"). As well as ex= \\ emplifying the meta-language, (yet) another definition \\ of ALGOL 60 is justified by the recent revision of the \\ language which resolved most of the open points in the \\ earlier "Revised Report".
}




\section{CONTENTS}

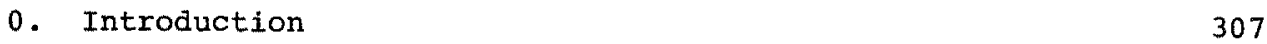

1. Abstract syntax 310

1.1 Definitions 310

1.2 Translator notes $\quad 312$

2. Context Conditions 313

2.1 "is-wf" Rules 314

2.2 "tp" Determining Rules 318

2.3 Auxiliary Functions $\quad 319$

$\begin{array}{ll}\text { 3. Semantic Objects } & 319\end{array}$

4. Meaning Functions 320

4.1 Functions from Object Language 320

4.2 Auxiliary Functions $\quad 334-336$ 


\section{O. INTRODUCTION}

For many years the official description of ALCOL 60 has been the "Revised Report" (Naur 63). Not only the language, but also its extreme1y precise description have been seen as a reference point. There were, however, a number of known unresolved problems and most of these have been eliminated by the recent modifications given in De Morgan 75 . A number of formal definitions exist for the language of the revised report: this paper presents a denotational definition of the language as understood from the modified report (MAR).

Before making some introductory remarks on the definition, three points will be made about the language itself (as in De Morgan 75). Firstly the modifications have followed the earlier "ECMA subset" by making the language (almost) statically typed. Although all parameters must now be specified, there is still no way of fixing the dimensions of array parameters nor the required parameter types of procedure or function parameters (cf. ALGOL 68). In connection with this it could be observed that the parameter matching rules of section 4.7 .5 .5 are somewhat difficult. In particular the definition given below assumes that, for "by name" passing of arithretic expressions, the types must match exactly:

The third observation is simply one of surprise. The decision to restrict the control variable of a <for statement> to be a <variable identifier> (i.e. not a subscripted variable) may or may not be wise: but the argument that <for statement> can now be defined by expansion within ALGOL is surely dangerous. The definition given here would have had no difficulty treating the more general case because the concept of location has anyway to be introduced for other purposes.

Two of the major points resolved by the modifications are the meaning of "own" variables and the provision of a basic set of input-output functions: particular attention has been given to these points in the formal definition below. In fact, the treatment of own given here is more detailed than that for PI/I static variables in Walk 69. Rather than perform name changes and generate dummy declarations in the outermost block, an extra environment component is used here to retain a mapping from (additional) unique names to their locations. This "Ownenv" is used in generating the denotations for own variables for insertion in the local "Env". The input-output functions are defined to change the "Channel" components of the state $(\Sigma)$. 
Much of the definition which follows should be easy to read after a study of the example given in Jones $78 \mathrm{a}$. The treatment of goto is similar to that given there (for discussion see Jones 75) except that the use of the "tixe" combinator has been held here to a minimum. Instead of the use in $i-b$ lock, an argument can be made for localizing the effects of goto at the level of comp-stmt, cond-stmt and stmt. In a version which used "tixe" at all three levels it was found advantageous to merge the "cue" and " $i$ " functions (cf. Jones 78b).

As has been discussed elsewhere in this volume, the definition of arbitrary order of evaluation has not been addressed: had it been, one would, for example, have to show that the elements of an expression can be evaluated in any order.

With the aid of the list of abbreviations given at the end of this introduction, the abstract syntax and context conditions should be straight forward. Notice that, although the abstract syntax itself is a "context-free" production system, context dependant typing (e.g. Arrayname) is used and secured by the context conditions. (Notice by-value variables are, in fact, non-by-name - i.e. by-value includes non-parameters).

The semantic objects are the key to the definition. States contain two components, one of which stores scalar values for each current scalar location (the division to the sub-types of $5 c-200$ is not necessary, it is only made to fit the implementation viewpoint), the other of which contains an abstraction of the objects which can be accessed by the input-output statements. State transformations are of the type required by the "exit treatment" of goto.

The composite objects Stmt-env and Expr-env are introduced solely as abbreviations; Own-env has been mentioned above; the real interest lies in the denotations which can be stored in Env. Type-dens are obviously scalar locations. A function procedure which is activated sets up a value to be returned by assigning to the Atv-proc-id: again a scalar location is the appropriate denotation. Array denotations store the (one-one) mapping from all possible subscript values to scalar locations; notice that the constraint requires that the domain of an array denotation is "dense". Procedure denotations are functions which, for given arguments and a current set of activations, yield transformations. Notice that the Act-parm-dens carry type information with them for checking within the Proc-den. Switch denotations are similar. 
The very general parameter passing "by name" permitted in ALCOL requires that the By-name-dens are rather like procedure denotations. Because formal parameter names can occur as Destinations for assignment statements it is also necessary to know whether a by-name parameter can be evaluated to a location or not (cf. e-parm-expr, e-varref, e-var). Furthermore, the question whether a parameter is to be passed by-name or by-value is not decidable at the point of call. Thus all parameters are passed by-name and the Proc-den has the task of creating the locations to store by-value parameters (cf. e-procdecl, e-val-parm).

The classes Loc and Val are auxiliary and are used only in type clauses.

Given an understanding of the semantic objects the reader should be able to tackle section 4. Remember that for "splitting" rules only a type clause is given.

\section{ACKNOWLEDGEMENT.}

Returning the compliment to Peter Mosses, one of the authors would like to acknowledge that a part of the incentive to write this definition was the hope to provide an equally abstract but more readable definition than that in Mosses 74 !

\section{ABBREVIATIONS.}

Abnormal component
actual
activation identifier
activated
arithmetic
assignment
by-name
Boolean
by-value
character
compound
conditional

constant
declaration
denotation
designator
descriptor
destination
element
environment
expression
function
identifier
integer

operator parameter procedure reference scalar specification statement subscripted transformation unzabelled value variable 


\section{ABSTRACT SYNTAX}

\subsection{Definitions}

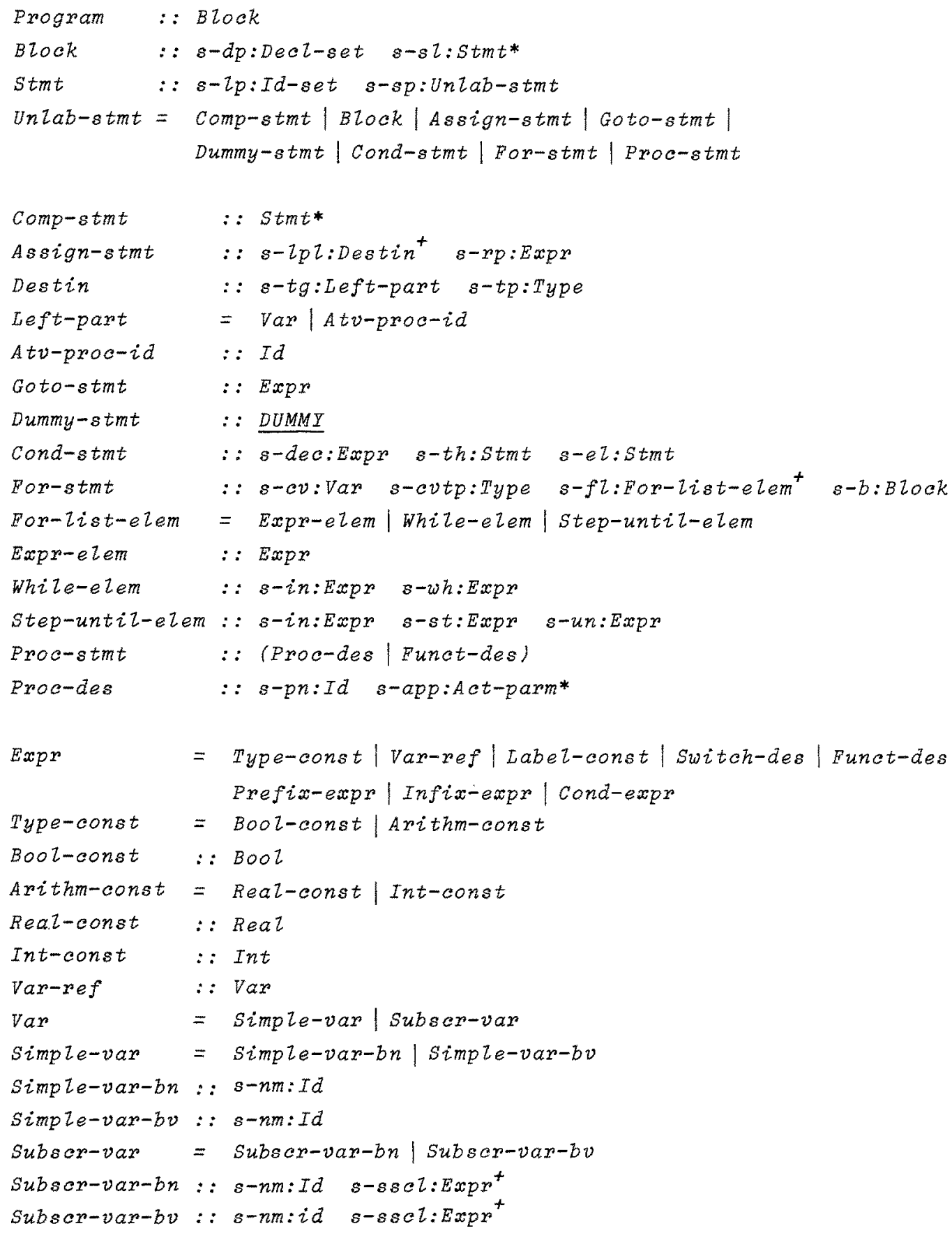




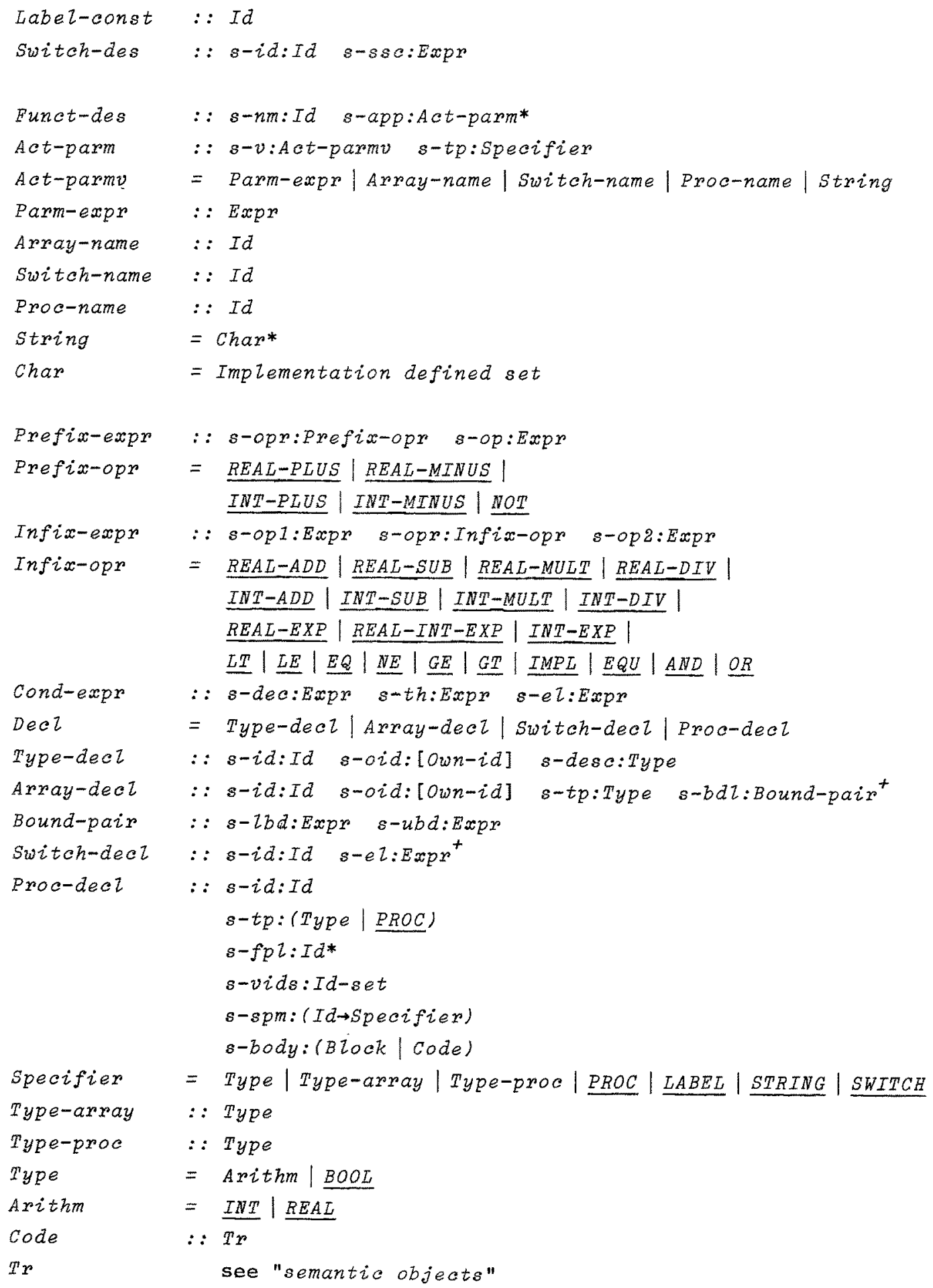




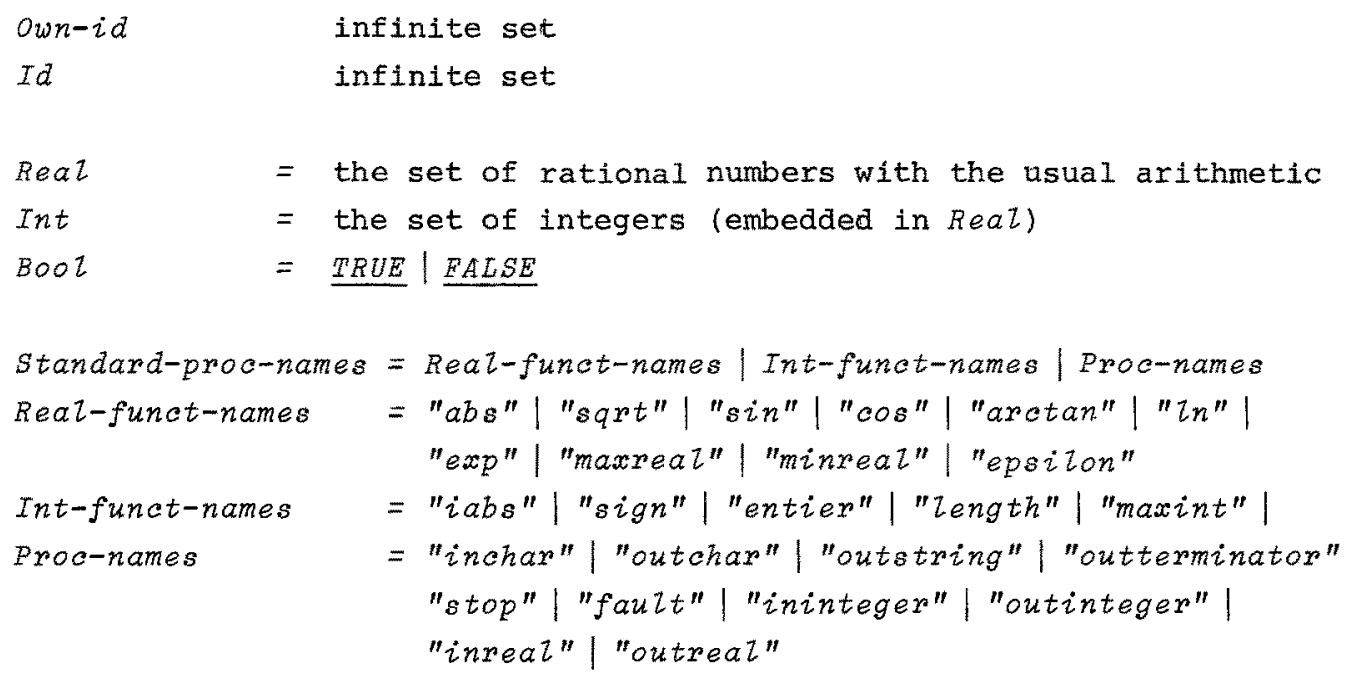

Comment: The quotes around the standard-procedure names indicate the translated version of the identifiers.

\subsection{Translator Notes.}

Although neither the concrete syntax of ALGOL 60 , nor its translation to objects of the abstract form are formally specified, a number of points should be borne in mind:

- Concrete delimiters, comments etc. are dropped.

- Within expressions, brackets and rules of operator precedence are used to choose the appropriate tree form of "expr".

- If the (concrete) outer block was labelled, the translator embeds it in another (unlabelled) block.

- The body of a procedure (which is not code) is always a block in the abstract form; the translator generates this block if it is not present in the concrete form.

- The body of a procedure which is code is translated into the appropriate state transformation. 
- Constants are, similarly, translated to (abstract) values.

- The outermost block (a created one, if necessary) contains the standard functions and procedures: where these cannot be expressed in ALGOL 60, meta-language descriptions of the transformations are given.

- The body of the abstract form of a for statement is always a block; if not present in the concrete form it is generated by the translator.

- The use of one <bourd pair list> to define several <array identifiexs $>$ is expanded by the translator. Notice that this can not be justified from MAR and, with side-effect producing function references in the bound pair list, is strictly wrong.

\section{CONTEXT CONDITIONS.}

An environment is used to record statically known type information:

$$
\text { Static-env }=I d \vec{m} \text { Specifier }
$$

With the exception of is-wf-program, all context conditions are, for a phrase class $\theta$, of type:

$$
i s-w f-\theta: \theta \text { Static-env } \rightarrow \text { Bool }
$$

As well as the splitting ("routing") rules, certain other obvious steps have been taken to shorten the functions given below, e.g. if

$$
\theta:: \theta_{1} \theta_{2} \ldots \theta_{n}
$$

then a rule (or part thereof) of the form:

$$
\begin{gathered}
i f-w f-\theta\left(m k-\theta_{1}\left(\theta_{1}, \theta_{2}, \ldots, \theta_{n}\right), e n v\right)= \\
i s-w f-\theta_{1}\left(\theta_{1}, e n v\right) \& \\
i s-w f-\theta_{2}\left(\theta_{2}, e n v\right) \& \ldots \\
i s-w f-\theta_{n}\left(\theta_{n}, e n v\right)
\end{gathered}
$$

will be onitted. 
2.1 is-wf Rules.

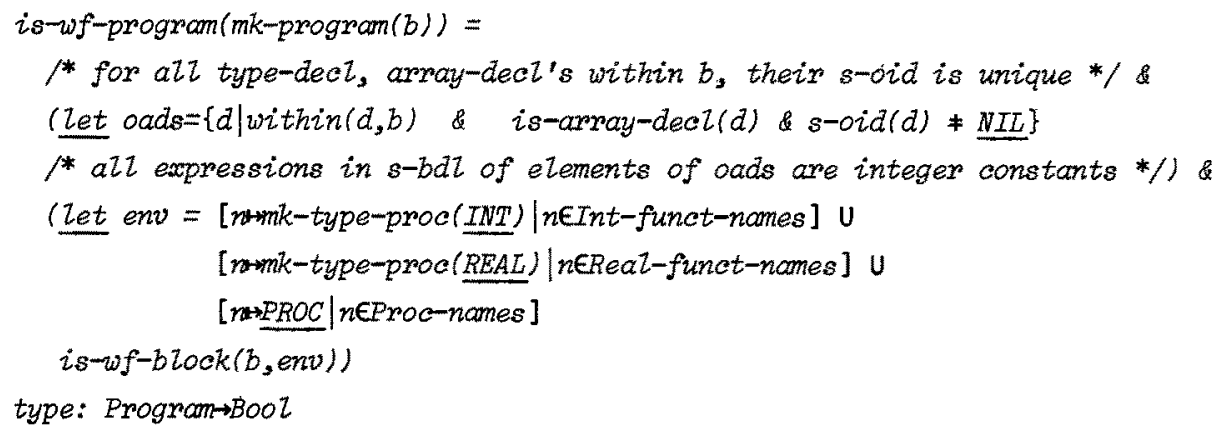


is-wf-assign-stmt (mk-assign-stmt $(d i, e), e n v)=$

$1 \leq i \leq l e n$ dr

(Zet $m k-\operatorname{destin}\left(Z_{p}, t p\right)=d L(i)$

compat-tps (tp, exprotp $(e, e n))$ \&

$\left(i s-\operatorname{var}\left(I_{p}\right) \Rightarrow\left(i s-s c a Z_{a r}\left(I_{p}, e n v\right) \&\right.\right.$

$t p=v a r-t p(t p, e n v))) \&$

$\left(i s-a t v-p r o c-i d\left(I_{p}\right) \Rightarrow t p=s-t y p e\left(e n v\left(I_{p}\right)\right)\right)$

$i s-w f-g o t o-s \operatorname{tmt}\left(m k-g o t o-s t m t(e)_{s} e n v\right)=\operatorname{expr-tp}\left(e_{,} e n v\right)=L A B E L$

$i s-\omega f-c o n d-s t m t(m k-c o n d-s t m t(d e c, t h, e l), e n v)=\exp p-t p(d e c, e n v)=B O O L$

is-wf-for-stmt (mk-for-stmt (ov, cvtp, flel, $b)$,env) $=$

is-simple-var(cv) \& is-scalar(cv,env) \&

is-arithm(cvtp) \& ovtp=var-tp(cv,env)

$i s-w f-\exp p-e l e m(m k-\exp -e Z e m(e), e n v)=i s-a r i t h m\left(\exp -t p\left(e_{,} e n v\right)\right)$

is-wf-while-eiem(mk-while-elem(in,wh),env) =

is-arithm(expr-tp(in,env)) \& is-BOOL(expr-tp(wh,env))

is-wf-step-until-eiem(mk-step-until-elem(in,st,un),env) =

is-arithm(expy-tp(in,env)) \&

is-axithm (expr-tp(st, env)) \&

is-arithm(expr-tp (un, env ))

is-wf-proc-des $(m k-p r o c-d e s(i d, a p i), e n v)=$ $i s-P R O C(\operatorname{env}(i d))$ \&

$(1 \leq i \leq \operatorname{len} \operatorname{apl} \Rightarrow s-\operatorname{tp}(\operatorname{apl} Z(i))=\operatorname{act}-\operatorname{parm}-\operatorname{tp}(s-v(\operatorname{ap} Z(i)), e n v)$

$i s-w f-v a r(v, e n v)=$

if is-simple-var(v) then is-type $(e n v(s-n m(v)))$

else is-type-array (env(s-nm(v))) \&

$(1 \leq i \leq \operatorname{len} s-\operatorname{sscl}(v) \Rightarrow i s-a x i t h m(\exp r-t p(s-s s c l(v)(i)$,env $)))$ 
$\left.\begin{array}{l}i s-w f-s i m p l e-v a a^{2}-b n / b v \\ i s-w f-s u b s c r-v a p-b n / b v\end{array}\right\}$ former iff refers to by name formal parameter

is-wf-label-const $(m k-i a b e l-c o n s t(i d), e n v)=e n v(i d)=\underline{L A B E L}$

is-wf-switch-des $(m k-s w i t c h-d e s(i d, e), e n v)=$

env $(i d)=$ SWITCE $\&$

is-arithm(expr-tp $(e, e n v))$

is-wf-funct-des $\left(m k-f u n c t-d e s\left(i d, \alpha_{p} z\right), e n v\right)=$

is-type-proc(env(id)) \&

$(1 \leq i \leq \operatorname{len} \operatorname{ap} I \Rightarrow s-\operatorname{tp}(\operatorname{apl}(i))=\operatorname{act}-\operatorname{parm} v-\operatorname{tp}(s-v(\operatorname{apl}(i)), \operatorname{env}))$

$i s-w f-a r r a y-n a m e(m k-a r r a y-n a m e(i d), e n v)=i s-t y p e-a r r a y(e n v(i d))$

is-wf-switch-name $(m k-s w i t c h-n a m e(i d), e n v)=e n v(i d)=$ SWITCH

$i s-w f-p r o c-n a m e(m k-p r o c-n o m e(i d), e n v)=i s-t y p e-p r o c(e n v(i d)) \vee \operatorname{env}(i d)=\underline{P R O C}$

is-wf-prefix-expr $(m k-p r e f i x-\exp r(o p r$, expr $)$, env $)=$

lett $t p=\operatorname{expr}-t p(\operatorname{expr}, e n v)$

(cases opr:

NOT

$\rightarrow t_{p}=\underline{B O O L}$

REAL-PLUS, REAL-MINUS $\rightarrow$ tp $=\underline{\text { REAL }}$

INT-PLUS, INT-MINUS $\rightarrow t p=\underline{I N T}$ 

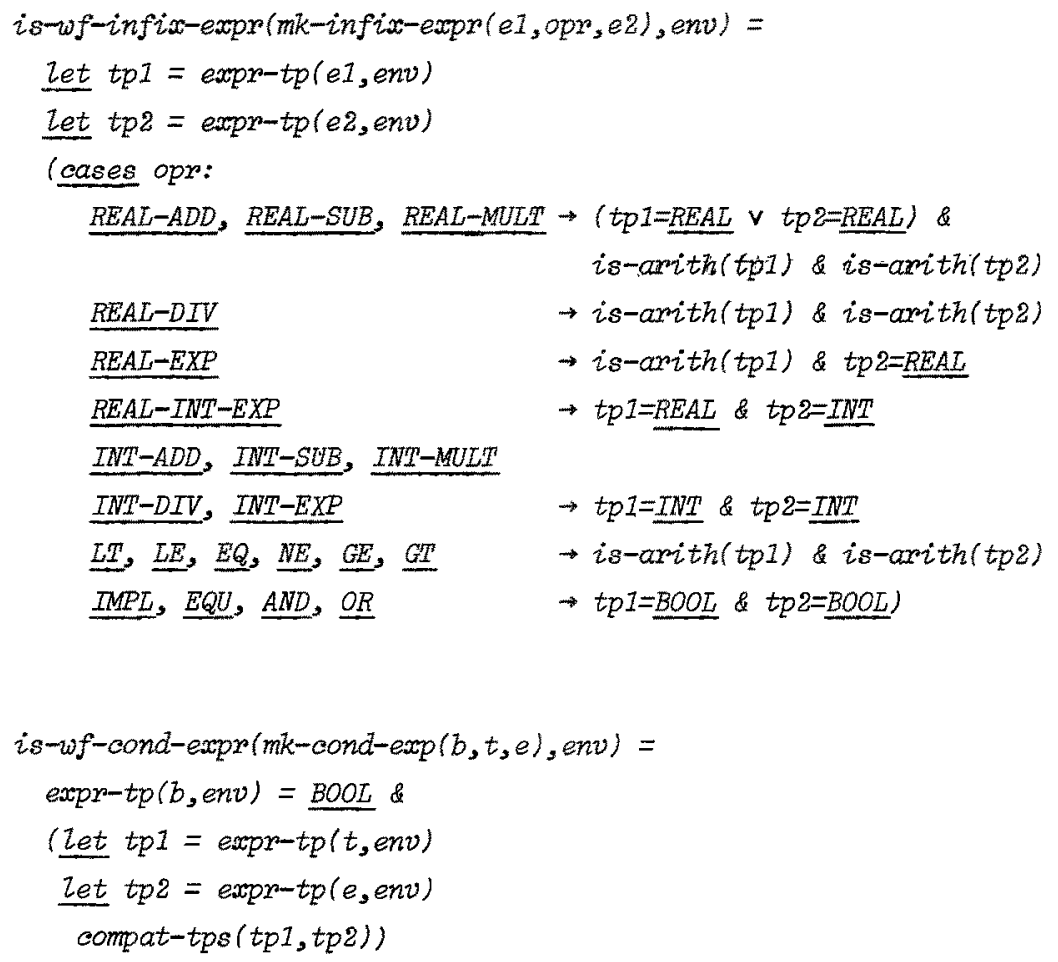

is-wf-array-deci(mk-array-decl(, tp, bdl), env) =

$1 \leq i \leq l e n b d l \Rightarrow i s-a x i t h(\operatorname{expr}-t p(s-l b d(b d l(i))$,env $) \&$ $i s-a x i t h(\operatorname{expr}-t p(s-u b d(b d i(i)), e n v)$

$i s-w f-s w i t c h-d e c l(m k-s w i t c h-d e c l(e x i), e n v)=$

$1 \leq i \leq l e n \operatorname{exl} \Rightarrow \exp x-t p(\operatorname{exl}(i)$, env $)=$ LABEL

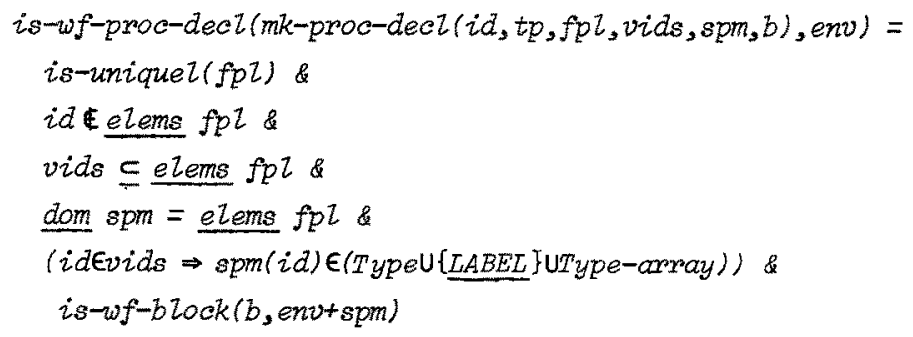




\section{2 tp Determining Rules.}

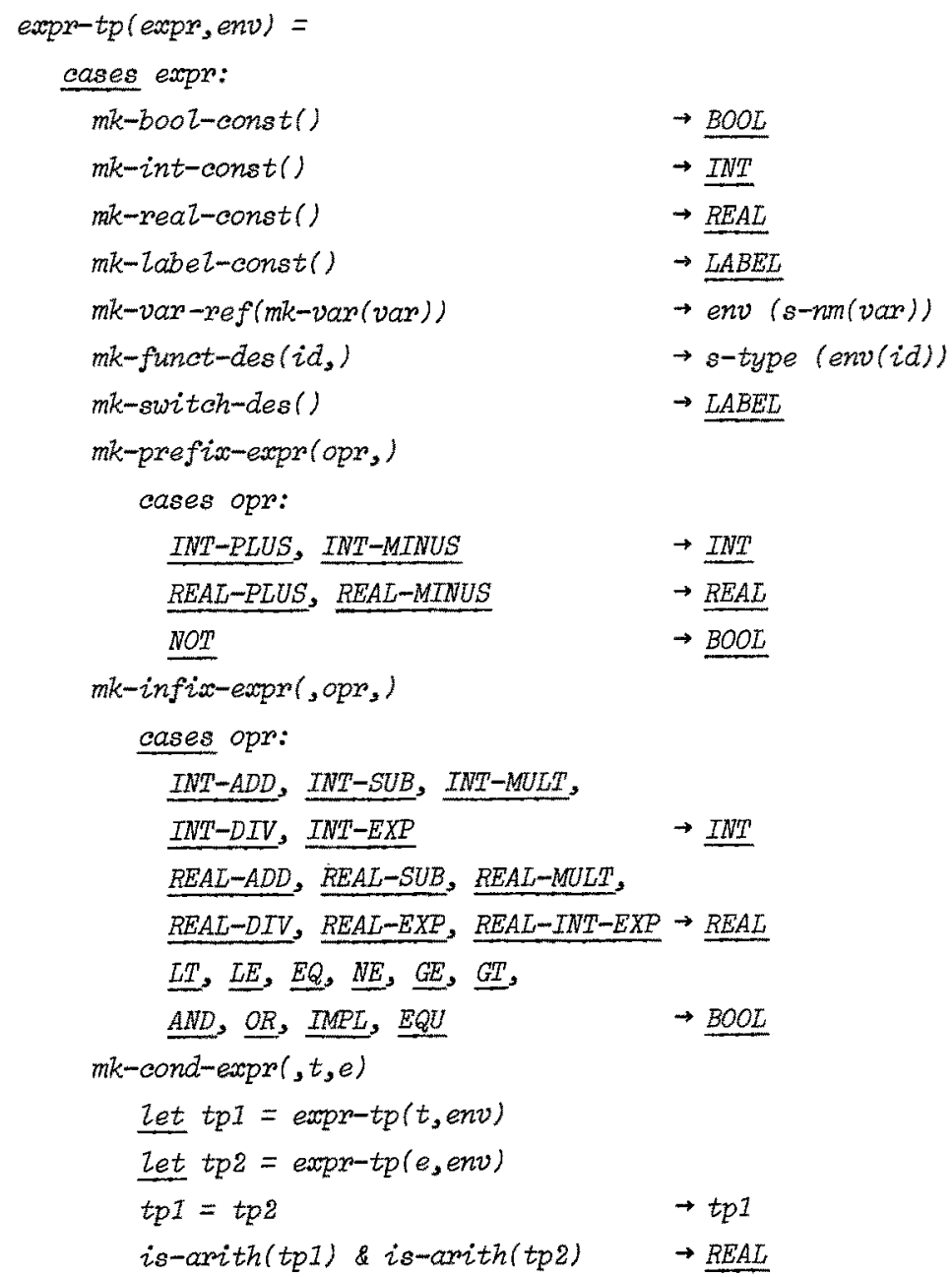

$v a r-t p(v, e n v s)=$

cases $v$ :

$m k$-simple-var $\left(i d_{2}\right) \quad \rightarrow \operatorname{envs}(i d)$

$m k-s u b s c r-v a r(i d$, sscl $) \rightarrow s-t y p e(e n v s(i d)$

act-parmv-tp similar to expr-tp 


\subsection{Auxiliary Functions.}

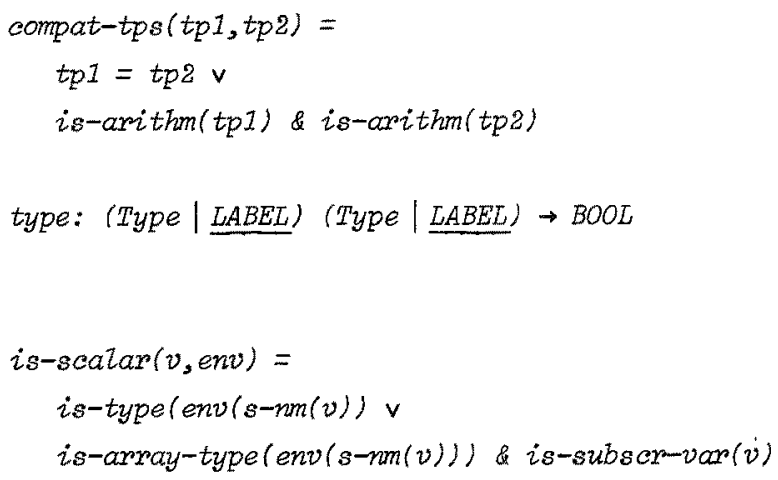

\section{SEMANTIC OBJECTS.}

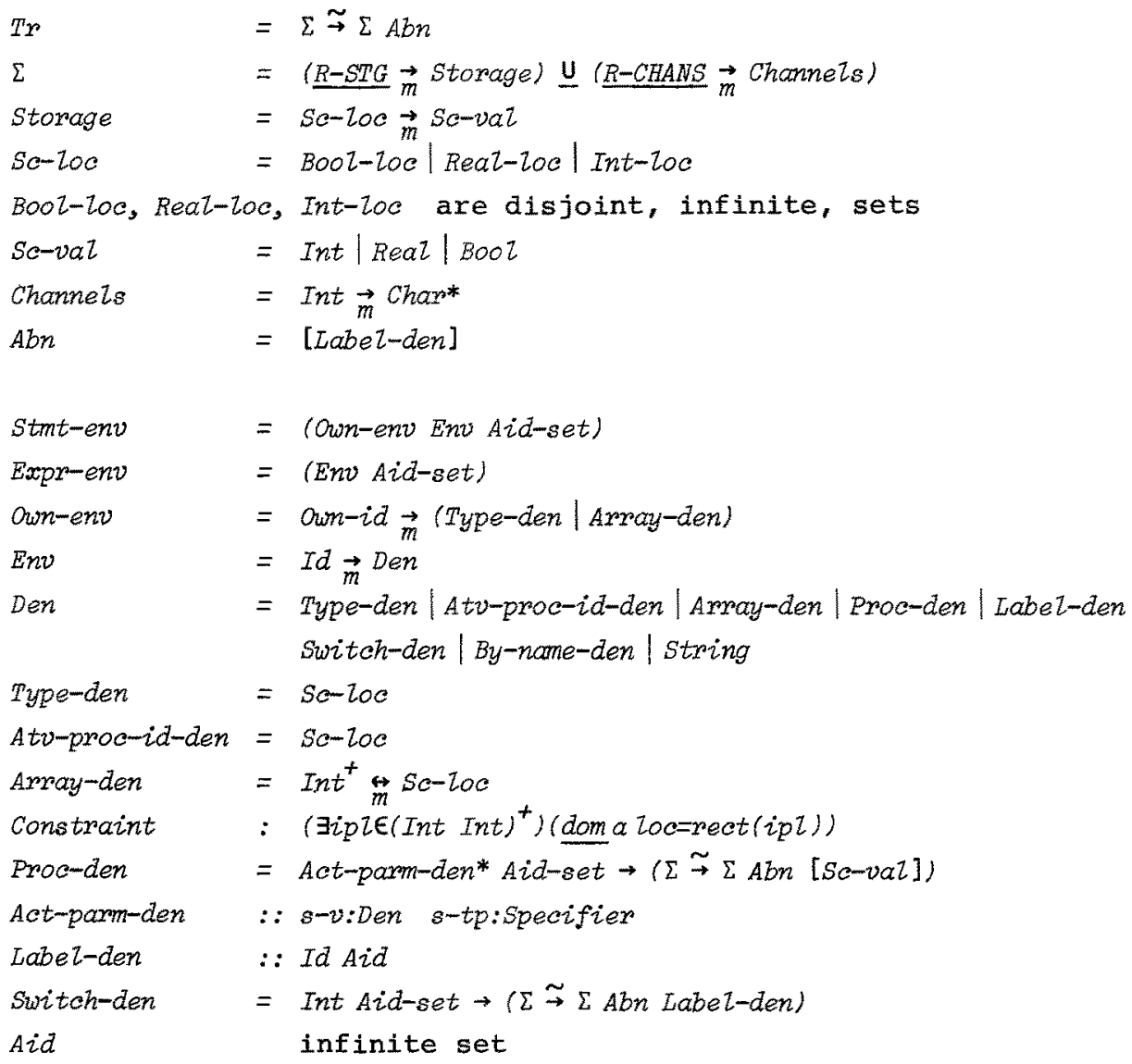




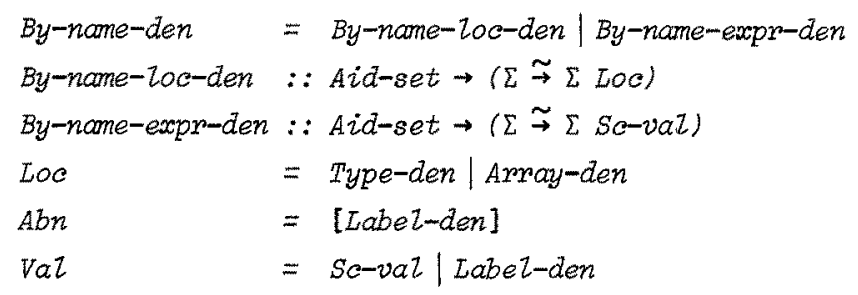

Implementation-defined-const $=$ MAXINT $\mid$ MINREAL $\mid$ MAXREAL $\mid \underline{\text { EPSILON }}$

Comment: The constants areSc-vals, but no check is made as to whether they are machine representable.

\section{MEANING FUNCTIONS}

\subsection{Functions from Object Language}

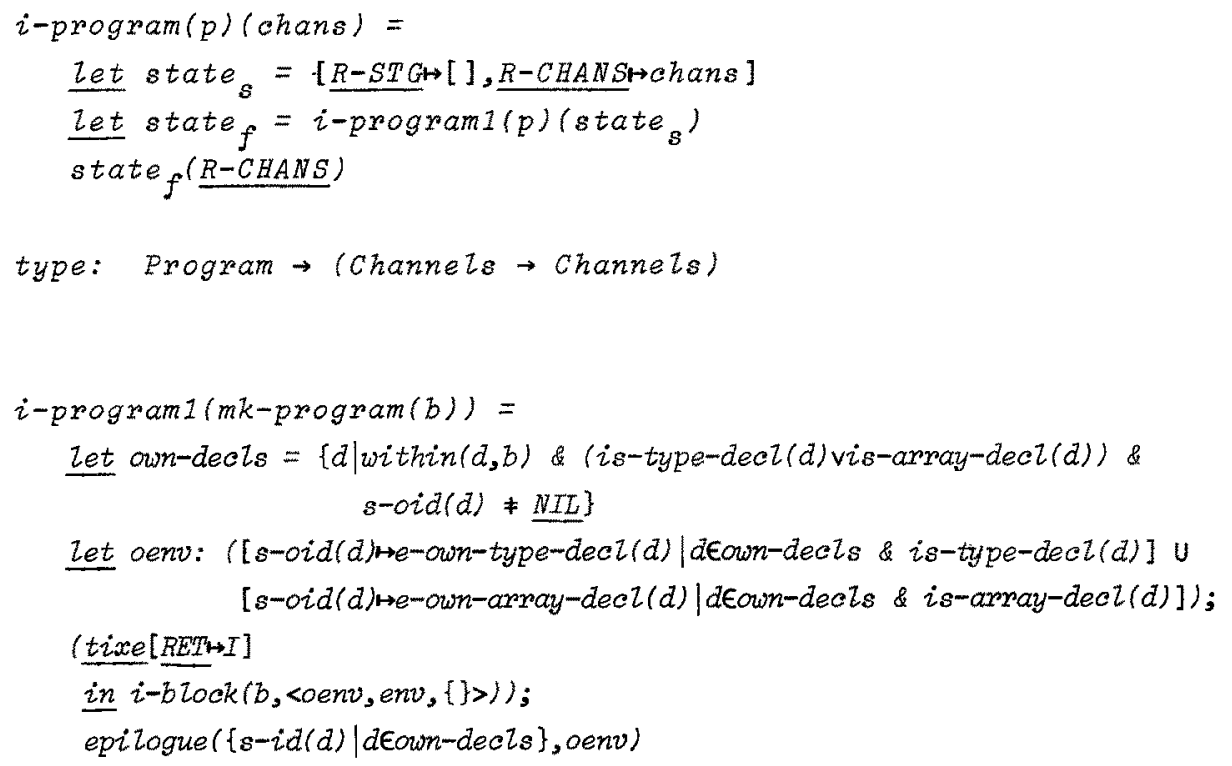




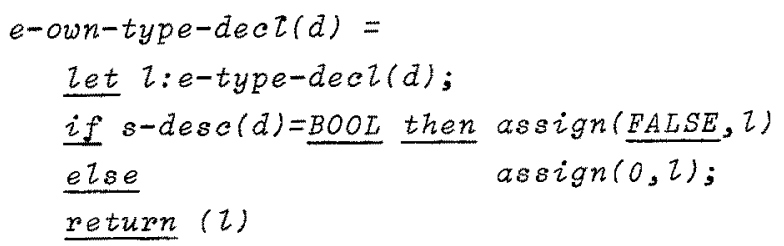

type: Type-dect $\Rightarrow$ Type-den

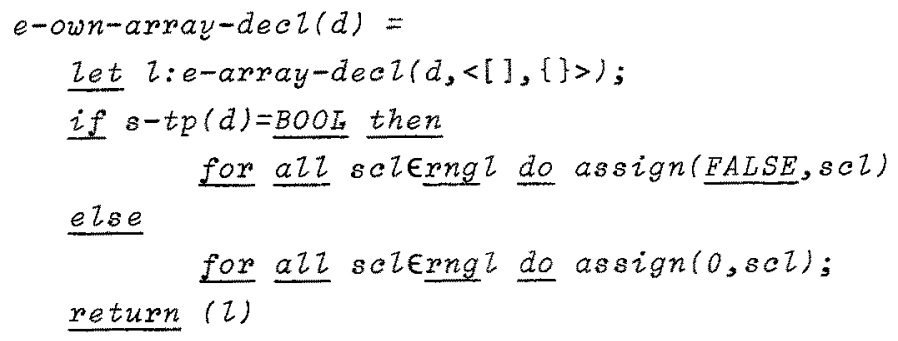

type: Array-dect Array-den

\section{Standard Functions and Transput}

It is assumed that the translation of the standard functions and procedures are contained in the ("fictitious") outer block. The interpre. tation of their proc-decl follows the normal interpretation rules (eproc-decl) except in the cases where the body cannot be expressed in Algol. In these cases the state transition of the non-Algol part is explicitly listed below.

Note: Referencing the translated identifiers we use quotes (e.g. "inreal" for the translation of the identifier inreal).

\section{In procedure stop:}

$$
\text { "goto } \Omega " \rightarrow \text { exit }(\underline{R E T})
$$


In procedure inchar: $\quad\langle$ body $\rangle \rightarrow$

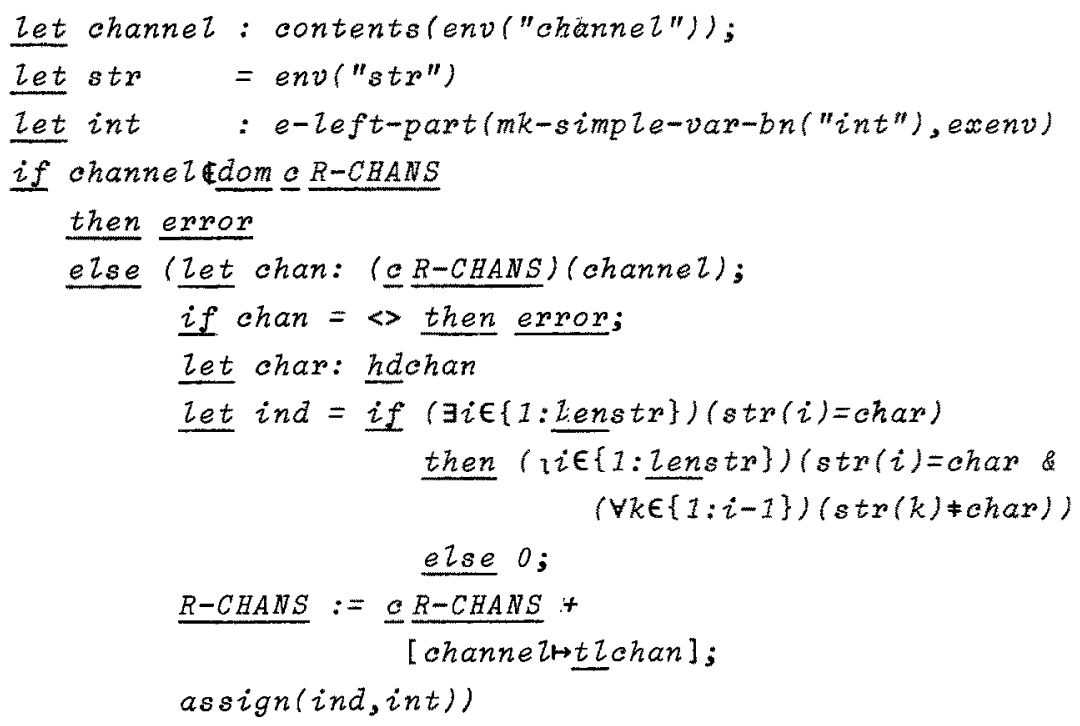

In procedure outchar: <statement> $\rightarrow$

Let channel : contents(env("channel"));

let $s t x=\operatorname{env}(" s t x ")$

Let int : contents(env("int"));

Let char $=\operatorname{str}(i n t)$

if channeldom $\underline{R}$-CHANS then error;

$\underline{R-C H A N S}::=\underline{e} \underline{R-C H A N S}+$

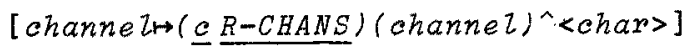

In procedure outterminator: $\langle$ body $\rangle \rightarrow$

Let channel : contents(env("channel"));

if channel $\$$ dom $\subseteq$ R-CHANS then error;

R-CHANS $\quad:=(\underline{c} \underline{R-C H A N S})+$

[channetr $(\underline{e} R-C H A N S)$ (channel)^<implementation

defined symbot depending on the current state of the channel>]

Procedures "maxint", "minreal", "maxreal" and "epsizon" have bodies which return the appropriate Implementation-defined-const. 


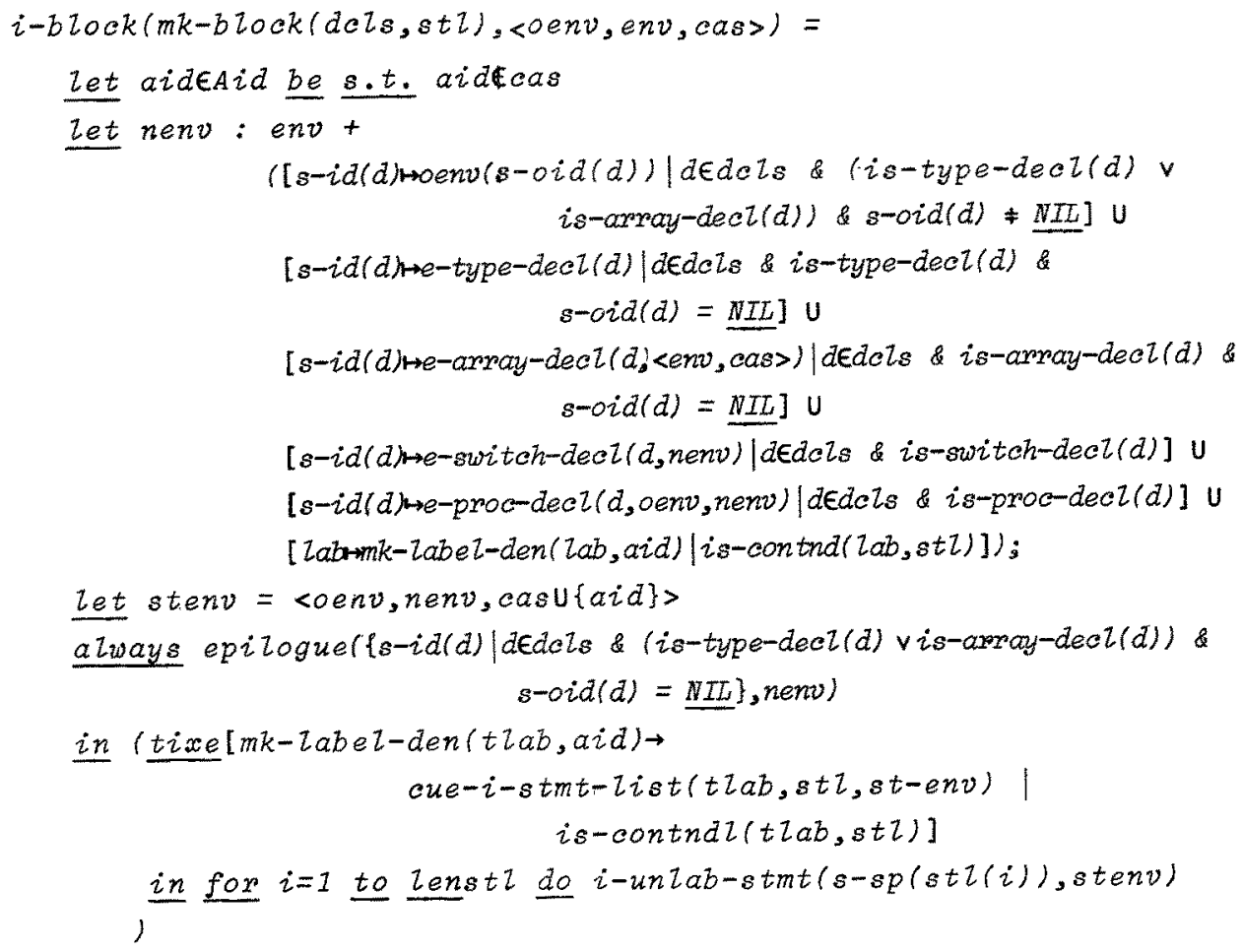

type: BZock Stmt-env $\Rightarrow$

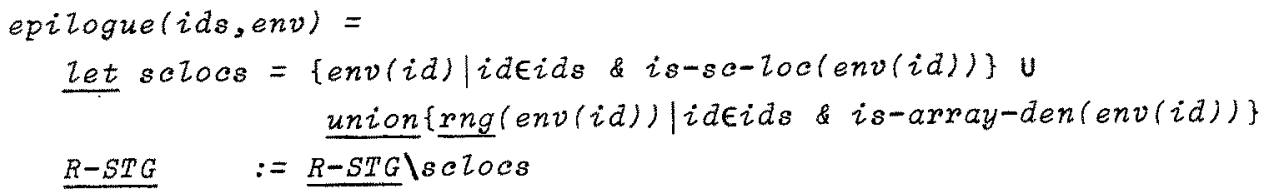

type: Id-set Env $\Rightarrow$

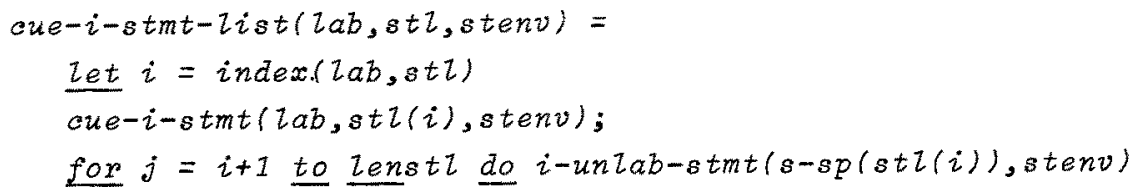

type: Id stmt*stmt-env $\Rightarrow$

pre: is-contndl( $2 a b, s t z)$ 
$c u e-i-s \operatorname{tm} t(2 a b, m k-s \operatorname{tm} t(2 a b s, s p), s \operatorname{tenv})=$

if zabElabs then i-unZab-stmt (sp, stenv)

else cue-i-unlab-stmt ( $a b, s p, s t e n v)$

type: Id Stmt Stmt-env $\Rightarrow$

pre: is-contnd ( $2 a b, m k-s t m t(2 a b s, s p))$

cue-i-unZab-stmt: Id UnZab-stmt Stmt-env $\Rightarrow$

cue-i-cond-stmt $(2 a b, m k-\operatorname{cond}-\operatorname{stm} t(, t h, e l)$, stenv $)=$

if is-contnd ( $2 a b$, th) then aue-i-stmt( $(a b, t h$, stenv)

else

cue-i-stmi ( $l a b, e l, s \operatorname{tenv})$

pre: $\quad$ is-contnd $(2 a b, t h) \vee i s-c o n t n d(2 a b, e l)$

cue-i-comp-stmt $(2 a b, m k-c o m p-s \operatorname{tm} t(\operatorname{st} \tau), \operatorname{stenv})=$ cue-i-stmt-iist( $2 a b, s t i, s t e n v)$

$i-u n Z a b-s t m t:$ UnZab-stmt Stmt-env $\Rightarrow$

$i-c o m p-s t m t(m k-c o m p-s t m t(s t z)$ stenv $)=$

for $i=1$ to lenst do $i-u n i a b-s t m t(s-\operatorname{sp}(s t i(i))$, stenv)

$i-a s s i g n-s t m t(m k-a s s i g n-s t m t(d \tau, e),\langle$, env, cas $\rangle)=$

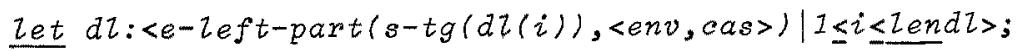

let $v: \quad e-\operatorname{expr}(e,<e n v, c a s>)$;

for $i=1$ to Zendi do

(Let vc:conv(v,s-tp(al(i)));

assign(ve,dz(i)))

e-teft-part: Left-part Expr-env $\Rightarrow S c-$ toc

$e-a t v-p r o c-i d(m k-a t v-p r o c-i d(i d),<e n v,>)=e n v(i d)$ 
$i$-goto-stmt (mk-goto-stmt $(e),\langle, e n v, c a s\rangle)=$

let $2 d: e-\operatorname{expr}(e,<e n v, c a s>)$;

exit (ld)

$i-d u m m y-s t m t(t, s t e n v)=I$

$i-\operatorname{cond}-s t m t(m k-\operatorname{cond}-s t m t(d e c, t h, e l)$, stenv $)=$

let $\langle$,env, cas> = st-env

let $b: e-e x p r(d e c,<e n v, c a s\rangle)$;

if $b$ then $i-u n l a b-s \operatorname{tmt}(s-s p(t h)$, stenv)

else $\quad i$-uniab-stmt $(s-s p(e l)$, stenv)

$i-f \circ r-s t m t(m k-f o r-s t m t(c v, c v t p, f l e r, b)$, stenv) $=$

for $i=1$ to Zenflel do $i$-for-list-elem (flez(i), cv, ovtp, $b$, stenv)

i-for-Zist-eZem: For-Zist-eZem Var Type Block Stmt-env $\Rightarrow$

$i-\exp r-e \operatorname{lem}(m k-\exp r-e \operatorname{lem}(e), c v, c v t p, b, s \operatorname{ten} v)=$

let $<$,env, cas> = stenv

let $v: e-\operatorname{expr}(e,<e n v, c a s>)$;

Let vc:conv (v,cvtp);

Let $Z: e-v a r(a v,<e n v, c a s>)$;

assign $(v e, z)$;

$i-b \operatorname{rock}(b, s \operatorname{tenv})$

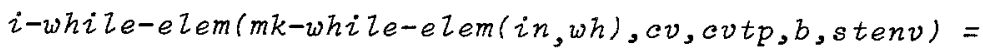

let $\langle$,env,cas> = stenv

while ( let $v: e-\exp r(i n,<e n v, c a s>)$;

let vc:conc $(v, c v t p)$;

let $Z: e-v a r(c v,\langle e n v, c \alpha s\rangle)$;

assign $(v e, z)$;

let $b: e-\operatorname{expr}(w h,<e n v, c a s>)$;

$b$ ) do $i-b \operatorname{rock}(b, s \operatorname{tenv})$ 


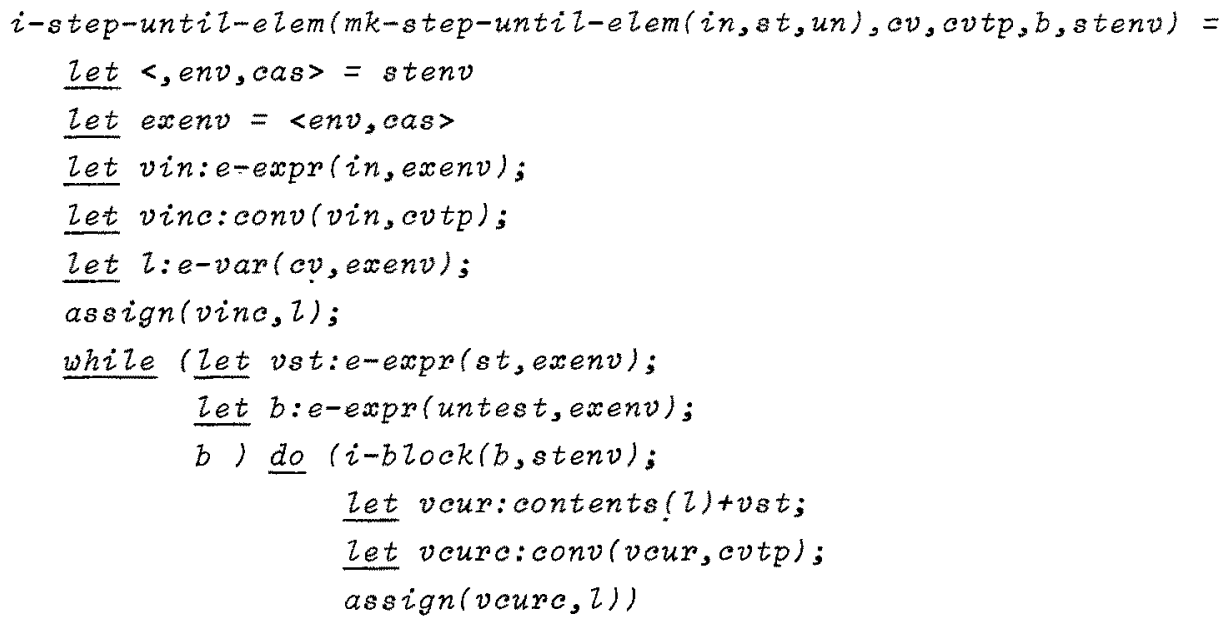

note: "untest" is an Expr corresponding to $\Gamma_{(\text {ev-un }) \times \operatorname{signvst} \leq 0}$

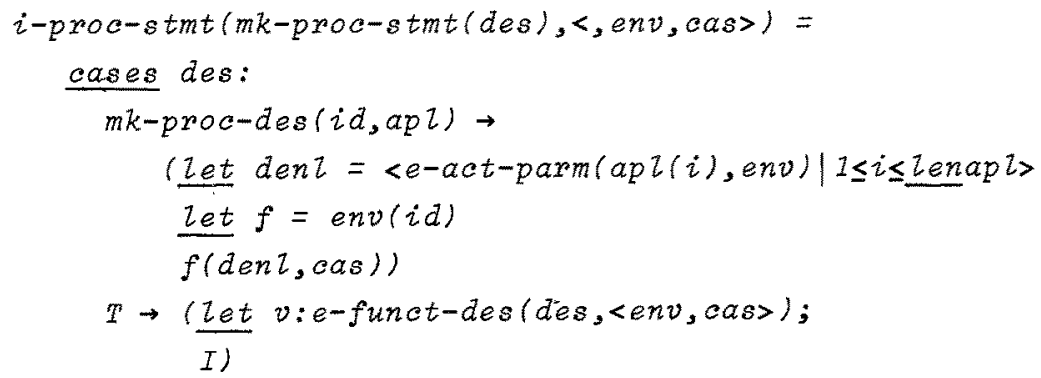

e-expr: Expr Expr-env $\Rightarrow$ Val

$e-b 0 o l$-const $(m k-b 0 o t-c o n s t(b))=,\operatorname{return}(b)$

$e-r e a l$-const $(m k-r e a l-c o n s t(r))=$, represent $(r)$

$e-i n t-c o n s t(m k-i n t-c o n s t(i))=$, test $(i)$ 
$e-v a r-r e f(m k-v a r-r e f(v),\langle e n v, c a s\rangle)=$

if is-simple-var-bv(v) $\vee i s-s u b s c r-v a r-b v(v) \vee i s-b y-n a m e-\operatorname{Loc}-\operatorname{den}(e n v(s-n m(v))$

then (Let $I: e-v a r(v,<e n v, c a s>)$;

contents( $(\tau))$

else (let bned $=\operatorname{env}(s-n m(v))$

bned(cas))

e-var: Var Expr-env $\Rightarrow$ Sc-Zoc

$e-s i m p l e-v a r-b n(m k-s i m p l e-v a r-b n(i d),\langle e n v, c a s\rangle)=$

let bnd $=\operatorname{env}(i d)$

if is-by-name-loc-den(bnd) then bnalcas)

ezse error

$e-s i m p l e-v a r-b v(m k-s i m p l e-v a r-b v(i d),<e n v,>)=e n v(i d)$

$e-s u b s c r-v a r-b n(m k-s u b s c r-v a r-b n(i d, s s c l),\langle e n v, c a s\rangle)=$

let esscl:e-subscrl(sscl, cenv, cas $)$ );

let $b$ ind $=\operatorname{env}(i d)$

if is-by-name-roc-den(bnd) then

(Let aloc:bnd(cas);

if esscledomatoo then return (alociessel))

else exror)

else exror

$e-s u b s c r-v a r-b v(m k-s u b s c r-v a r-b v(i d, s s c l),\langle e n v, c a s\rangle)=$

let esscl:e-subscrl(sscl, <env, cas>);

Let $a$ loc $=\operatorname{env}(i d)$

if esscledomaloo then return (aloc(esscl))

else exror 
e-subscrl (sscl, exenv) =

Let essol: <( let essc:e-expr(sscl(i), exenv);

let $i: \operatorname{conv}(\operatorname{essc}, \underline{I N T})$;

$i \quad \| 1 \leq i \leq$ lenssci>;

return (essel)

type: Expr* Expr-env $\Rightarrow$ Int*

$e-2 a b e l-c o n s t(m k-2 a b e l-c o n s t(i d),<e n v,>)=$ return $(e n v(i d$,

$e-s w i t c h-d e s(m k-s w i t c h-d e s(i d, s s c),\langle e n v, c a s\rangle)=$

let ess:e-expr(ssc, <env, cas>);

let $i: \operatorname{conv}(e s s, I N T)$;

Let $f=\operatorname{env}(i d)$

Let $2 d: f(i, c a s)$;

return $(i d)$

$e$-funct-des $(m k-f u n c t-d e s(i d, a p l),\langle e n v, c a s\rangle)=$

let denl $=\langle e-a e t-p a r m(a p l(i)$,env) $\mid 1 \leq i \leq$ lenapl $\rangle$

Let $f=e n v(i d)$

Let $v: f(d e n z, c a s)$;

return (v)

$e-a c t-p a r m(m k-a c t-p a r m(e, t p), e n v)=$

Let $d=e-a c t-p a r m v(e, e n v)$

$m k-a c t-p a r m-d e n(a, t p)$

type: Act-parm Env $\rightarrow$ Act-parm-den

e-act-parmv: Act-parmv Env $\rightarrow$ Den 


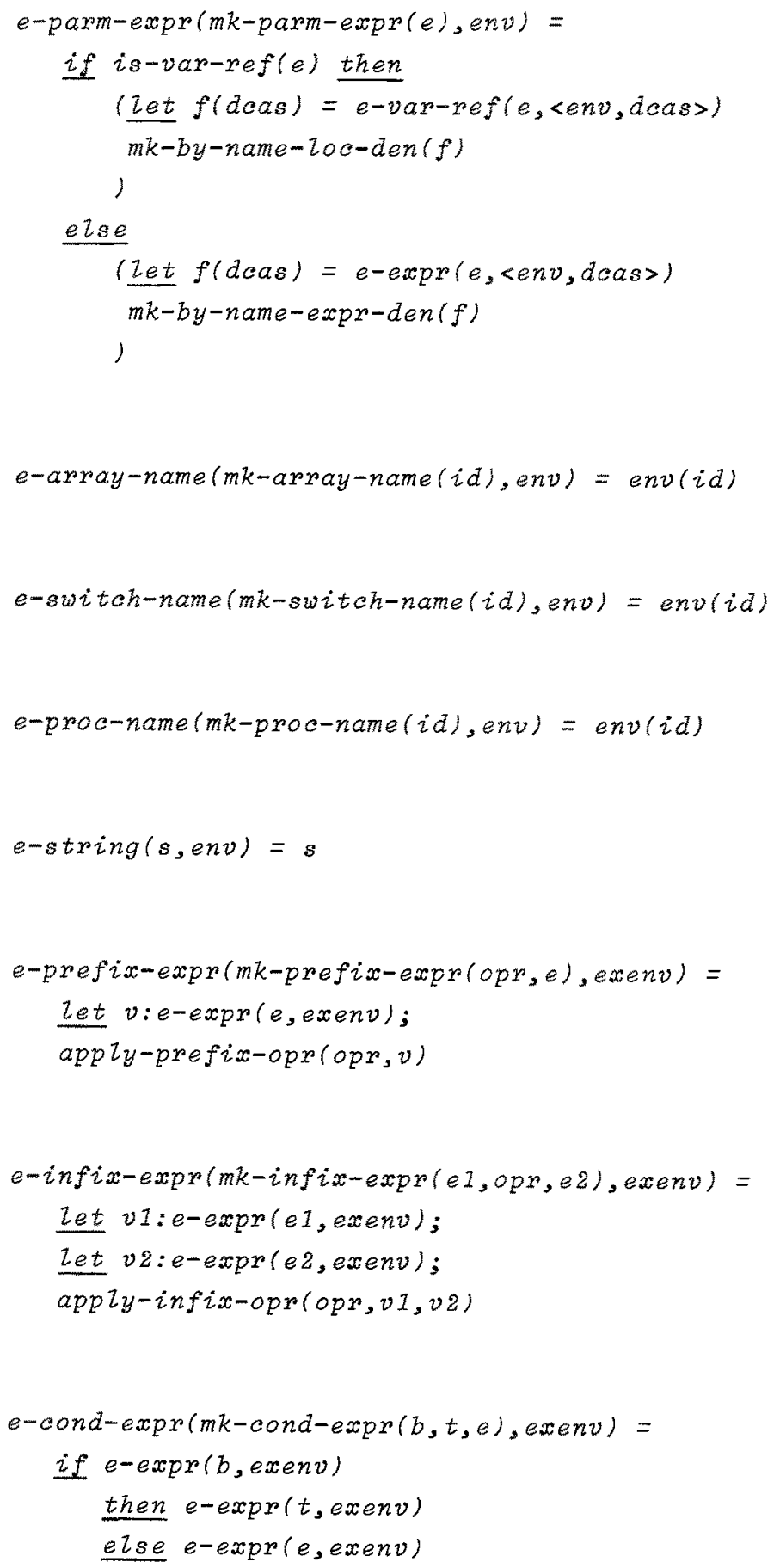


Comment: The evaluation of infix expressions is from left to right. since IntçReal no explicit conversion from integer to real is necessary in infix and conditional expressions.

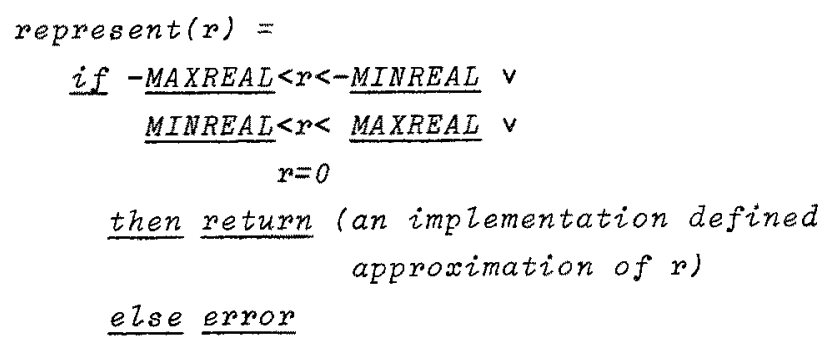




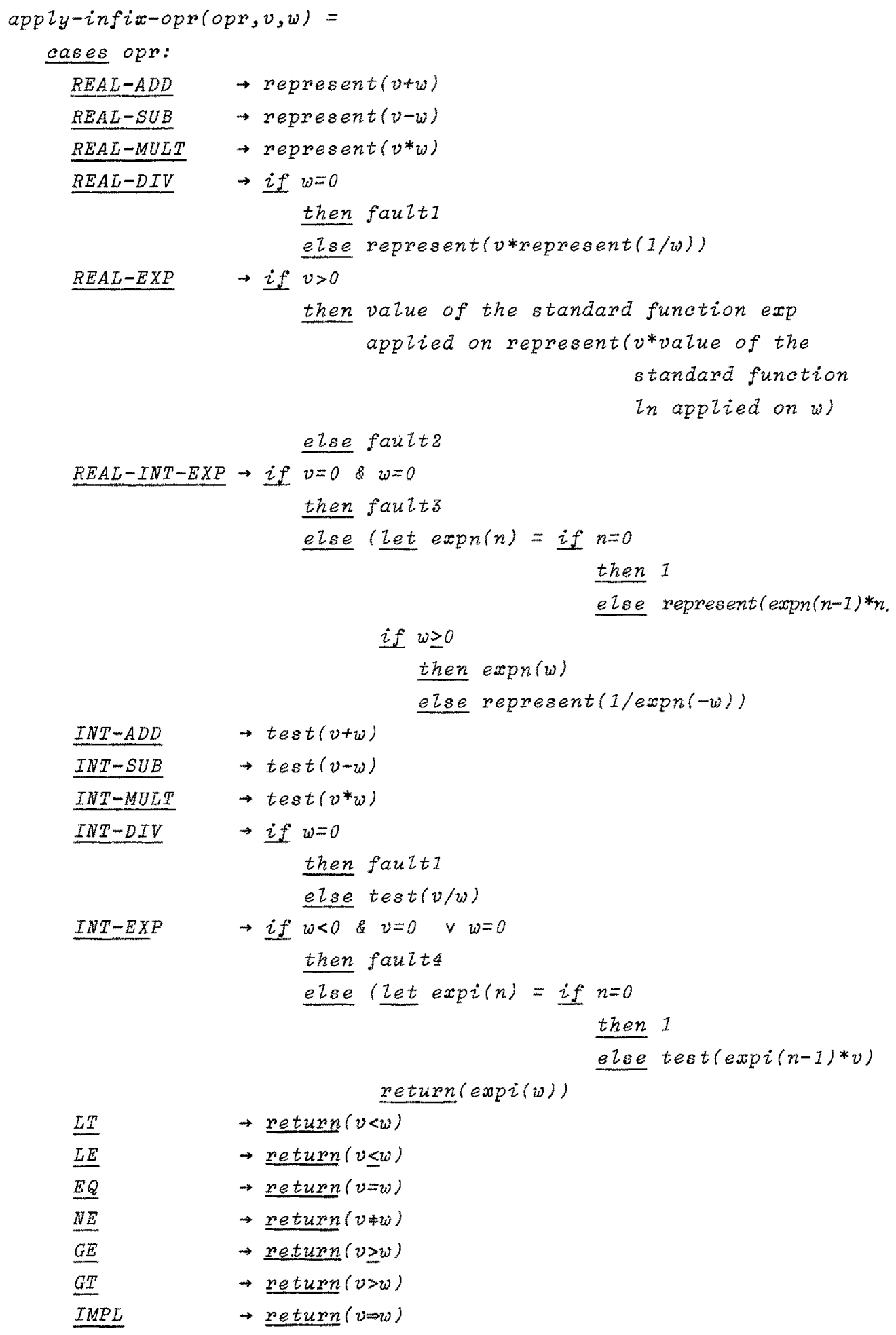




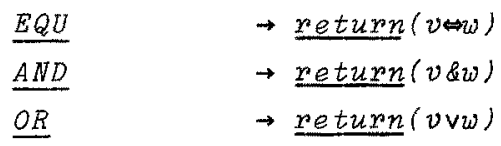

type: Infix-opr Sa-val Se-val $\Rightarrow S e-v a l$

Comment: fauttl represents the state transition, which corresponds to the call: fautt('div by zero',v)

faultz $\sim$ fault ('expr undefined',v)

fault3 fault('expn undefined', $v$ )

faults $\sim$ fault ('expi undefined',w)

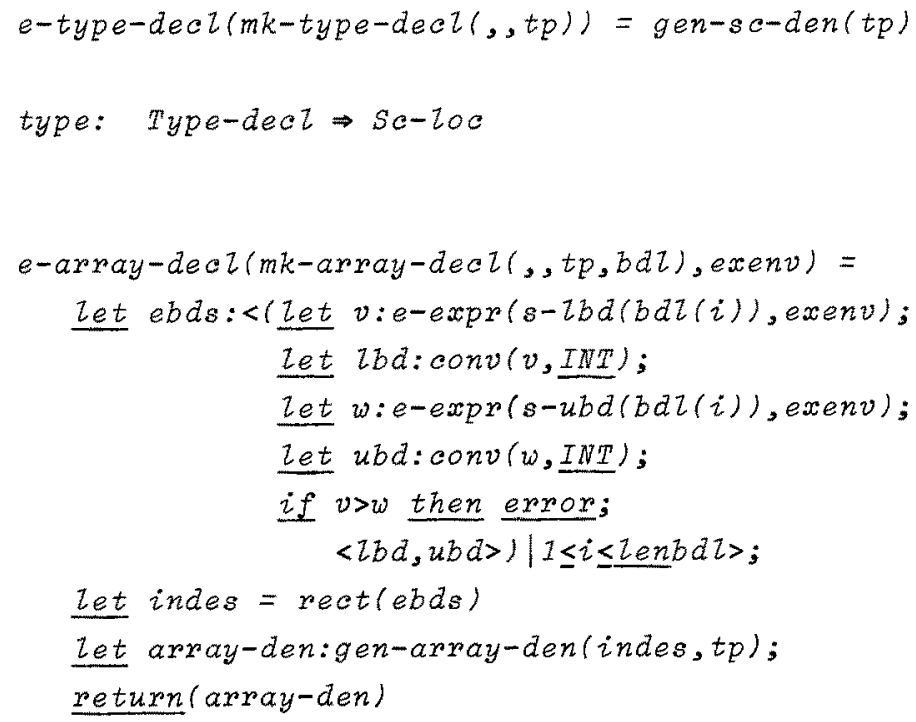

type: Array-decl Expr-Env $\Rightarrow$ Array-den

Comment: It is assumed that the generation of an array without elements is exroueous, rather than the access to such an array. 


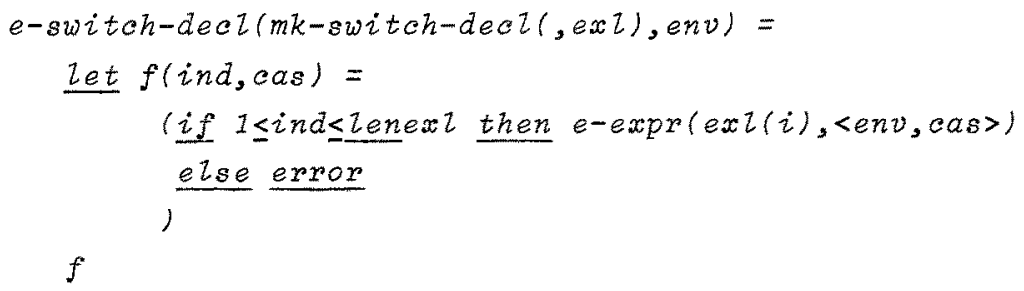

type: Switch-decl Env $\rightarrow$ Switch-den

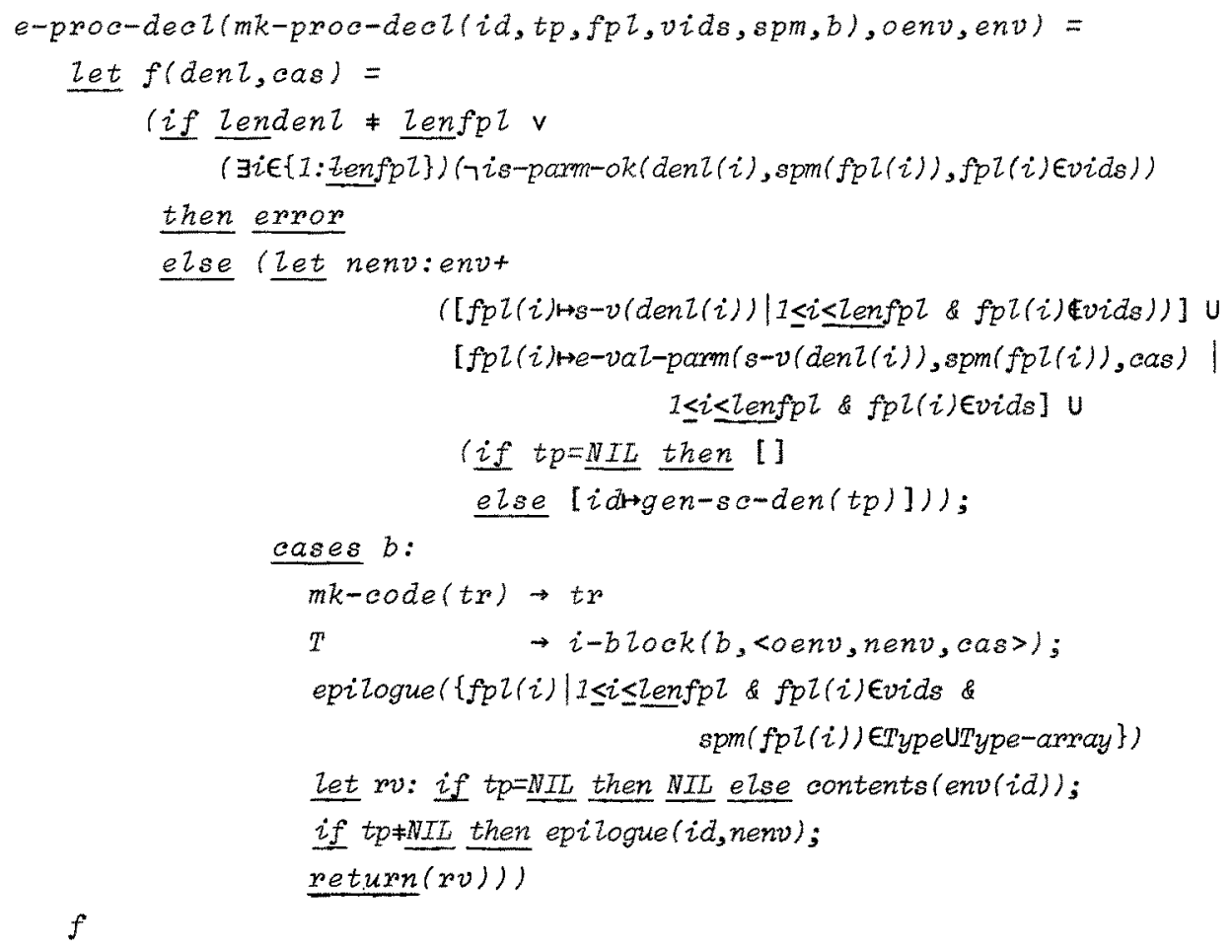


is $-p a r m-o k(\langle v, s p a\rangle, s p f, b v)=$

if $b v$ then

( spa=spf $\vee i s-a r i t h m(s p f) \& i s-a r i t h m(s p a) \vee$

is-type-array (spf) \& is-type-array (spa) \& is-axithm(s-type(spa))

\& is-arithm(s-type (spf)))

else

$(s p a=s p f \vee s p f=P R O C$ \& is-type-proc(spa) $\vee$

is-type-proc(spf) \& is-type-proc(spa) \& is-arithm(s-type(spa))

\& is-arithm(s-type(spf)))

type: Act-parm-den Specifier Bool $\rightarrow$ Bool

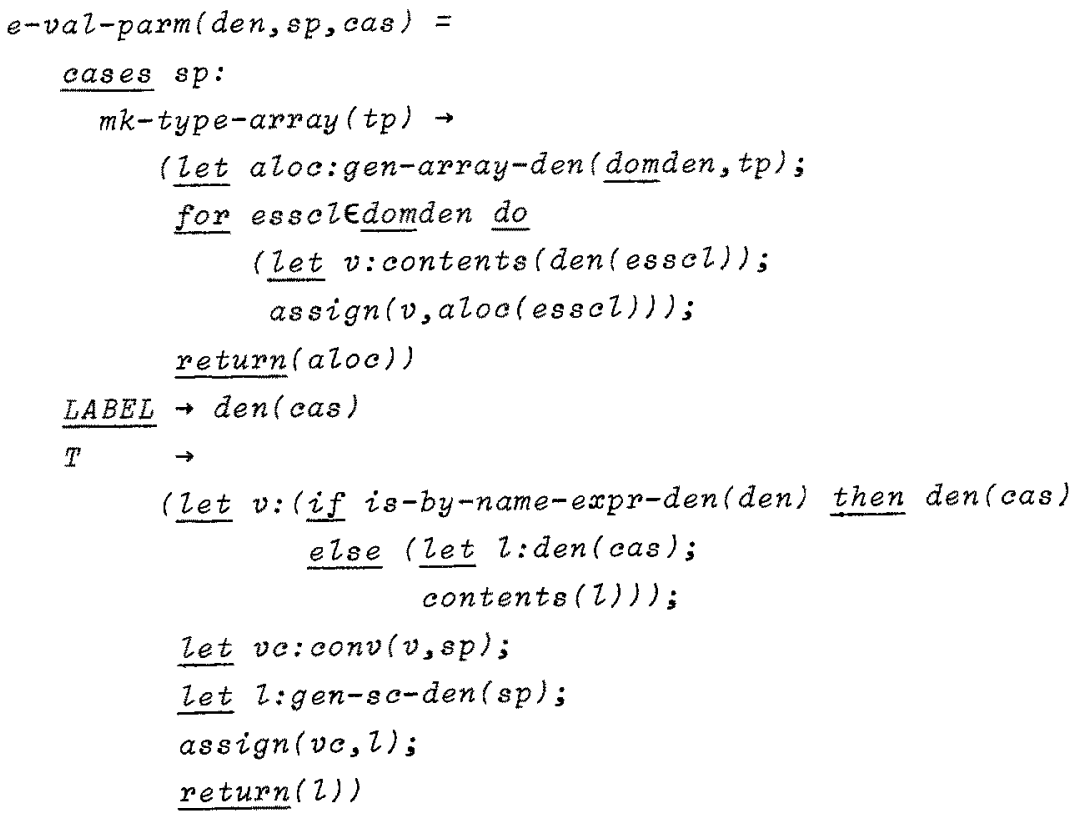

type: Den Specifier Aid-set $\Rightarrow$ Den

\subsection{Auxiliary Functions}

is-contnd: Id Stmt $\rightarrow$ Bool

is-contndl: Id Stmt* $\rightarrow$ Bool

Comment: Two obvious functions for checking whether the given identifier is contained in the label part of a (contained) statement which is not contained in an intervening block. 
index: Id Stmt* $\underset{\sim}{\rightarrow}$ Nat

Comment: For identifiers which satisfy "is-contndl" this function finds the index such that the indexed element of the state ment list also contains the identifier.

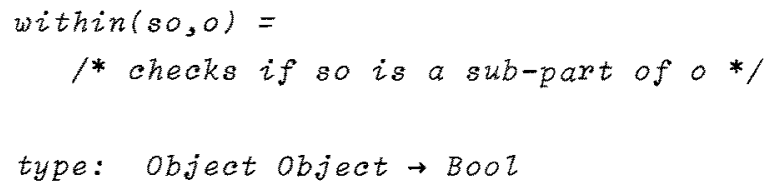

Comment: True iff no duplicates

is-disjoint: (Object-set)* $\rightarrow$ Bool

Comment: True iff sets are pairwise disjoint

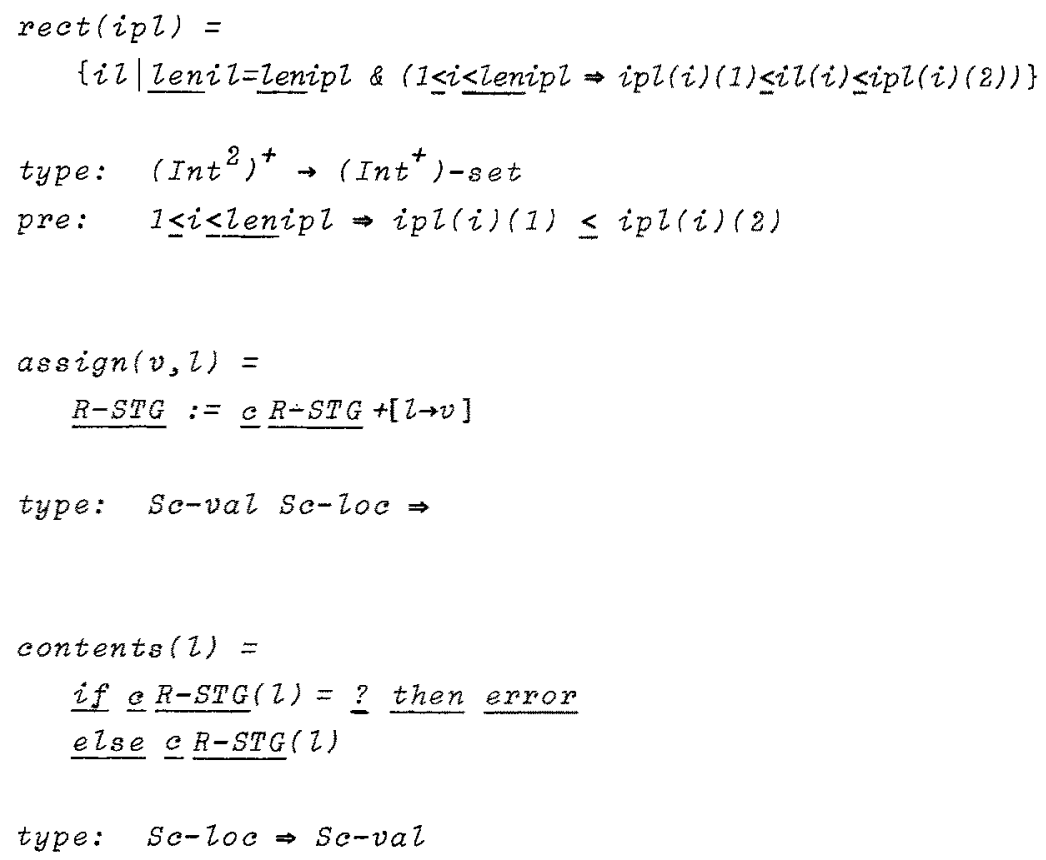


